\title{
ARTICLE
}

\section{Smartphone apps in mental healthcare: the state of the art and potential developments ${ }^{\dagger}$}

\author{
Melvyn W. B. Zhang, Cyrus S. H. Ho, Christopher C. S. Cheok \& Roger C. M. Ho
}

\begin{abstract}
Melvyn Zhang is a 4th year resident with the National Healthcare Group Singapore and is currently working with the Institute of Mental Health, Singapore. He has a special interest in the application of information technologies in psychiatry. Cyrus $\mathbf{H o}$ is a specialist registrar at the National University Hospital, Singapore. His special interests include neuropsychiatry, neurorehabilitation and the interface between medicine and psychiatry. Christopher Cheok is the Vice Chairman of the Medical Board (National Addictions Management Service) at the Institute of Mental Health. He is a visiting consultant to the Singapore Armed Forces and an advisor to the Juvenile and Family Court of Singapore. He is also a member of the medical board of the Civil Aviation Authority of Singapore. He has a special interest in child and adolescent psychiatry, psychological trauma and research. Roger $\mathbf{H o}$ is an assistant professor and consultant psychiatrist at the National University Hospital, Singapore. He has a special interest in psychoneuroimmunology and the interface between medicine and psychiatry. Correspondence $\mathrm{Dr}$ Melvyn Zhang Weibin, National Addictions Management Service (NAMS), Institute of Mental Health, 10 Buangkok View, Buangkok Green Medical Park, Singapore 539747 Email: melvynzhangweibin@gmail. com
\end{abstract}

tFor a related commentary, see pp. 359-360, this issue.

\begin{abstract}
SUMMARY
Previous studies have demonstrated that smartphones are useful tools in everyday, evidence-based medical practice. This article gives an overview of the current use in psychiatry of smartphone apps aimed at patients and the general public, highlighting associated benefits and disadvantages. It also outlines how practising psychiatrists could embrace such technologies at an individual, organisational and national level.
\end{abstract}

\section{LEARNING OBJECTIVES}

- Recognise the current uses of smartphone technologies in psychiatry and their inherent advantages and disadvantages

- To empower practising psychiatrists to create evidence-based mental health apps for patient care

- Understand how smartphone technologies could be used at an organisational and a national level to add value to current service provision

\section{DECLARATION OF INTEREST}

M.W.B.Z. designed and created the Royal College of Psychiatrists' Mental Health Key Facts app. The creation of this app is supported by the College's Public Education Engagement Board, and the author received no payment for this work.

As we discussed in the previous issue of Advances (Zhang 2015a), smartphones are not just communication devices: their ability to access the internet and to run quite complex software programs (applications or apps) makes them computers in miniature. It is not surprising to learn of the high rates of adoption of smartphones by healthcare professionals: the highly mobile work of clinicians in the present-day healthcare system, embracing the clinic, the ward, the specialist out-patient service, the emergency department, the laboratory and many other locations, makes a smartphone invaluable (Mosa 2012). There has also been an increase in the number of software programs developed specifically to cater to the needs of clinicians, in particular the launch of more evidence-based smartphone apps (Marshall 2008). The clinical utility of such apps in the medical disciplines has been well established, with numerous recent publications about smartphone interventions in medicine (see Zhang 2015a). Current uses of smartphone technologies for patient care in medical disciplines include apps for the management of chronic disease (Mosa 2012; Ozdalga 2012) and interventional tools for stroke and cardiac rehabilitation programmes (Edgar 2010; Worringham 2011). Smartphones have also been used as monitors to facilitate medical and clinical diagnosis of conditions such as sleep apnoea (Bsoul 2011), as electrocardiogram recording devices (Oresko 2010) and as Doppler devices for measuring blood flow (Huang 2012).

\section{Apps for patients' use}

We carried out an extensive search of the literature on the use of smartphone apps intended for patients' use in mental healthcare, and found that few randomised controlled trials have been conducted to evaluate those that are available. We discuss some of the studies that we did find.

\section{Psychosis and schizophrenia}

Palmier-Claus et al (2012) evaluated a smartphone app that prompts users to complete a self-report questionnaire on current psychotic symptoms. They found that the app was a feasible and valid way of assessing symptoms and that it accurately correlated the patient's symptoms with stressors. This in itself would help psychiatrists to identify specific stressors that lead to an early relapse in individuals who are capable of reporting their symptoms. In a qualitative study of smartphonebased assessment using both an app and text messaging, the same research team found that selfmonitoring of psychotic symptoms made patients more aware of them (Palmier-Claus 2013). The team suggested that such interventions might also improve the doctor-patient relationship, as the psychiatrist would have a better understanding of what the patient had been experiencing. 


\section{Affective disorders}

Studies also demonstrate the clinical utility of smartphones in bipolar disorder, depression and anxiety. Grünerbl et al (2015) found that smartphone apps coupled with wearable sensors were effective in identifying changes in the affective state of patients with bipolar disorder. In another study involving people with bipolar disorder, Faurholt-Jepsen et al (2014) demonstrated that a smartphone app could help individuals to monitor their depressive symptoms and recognise how these could affect their physical functioning.

Similar findings were obtained by BinDhim et al (2015), who showed the clinical effectiveness of a smartphone app that included the nineitem Patient Health Questionnaire (PHQ-9) as a screening tool for depression.

Research has also demonstrated that patients benefited from a smartphone app that equipped them with skills pertaining to coping with their underlying anxiety condition (Pramana 2014).

\section{Substance misuse}

A study on a smartphone app provided to patients as an adjunct to face-to-face therapy sessions for substance use (Rizvi 2011) found that the app reduced their craving to use substances.

\section{Dementia}

The efficacy of smartphone apps is not limited to patients who are technologically savvy. Sposaro et al (2010) reported that a smartphone's built-in Global Positioning System (GPS) receiver could be used to monitor the location of people with Alzheimer's dementia who are at risk of wandering off from their usual setting.

\section{Strengths and limitations of available apps}

\section{Strengths}

Psychiatry apps offer multiple benefits for patient care (Box 1). They could be used to enable individuals to take greater 'ownership' of their

\section{BOX 1 Some benefits of apps for mental} healthcare

- Patients can acquire 'ownership' of their psychiatric condition

- Psychoeducation of patients, family and carers

- Identification of stressors that precipitate relapse

- Greater insight of psychiatrist into patient's mental state

- Adjunctive tools in time-limited psychotherapy psychiatric condition, by allowing them to monitor their symptoms. The inclusion of appropriate resources in the app can help to educate individuals, family and carers about mental health conditions. Apps for symptom self-monitoring can help psychiatrists to identify stressors that might precipitate a patient's early relapse and also to intervene early to prevent a full relapse. Empowering individuals with self-monitoring tools might add value to the psychiatrist-patient relationship, as the psychiatrist would be able to gain greater insight into the well-being of their patients: rather than relying solely on collateral information from families or a short consultation with the patient, the psychiatrist might learn much from the self-reports captured on the app. Apps can also be used as adjunctive tools to augment a time-limited psychotherapy intervention.

There are other potential benefits of smartphone apps for patient care that have not been clearly identified. Our discussion apps is largely limited to published data, but there are many apps that have undergone no formal rigorous evaluation of clinical effectiveness. Apps such as pill-trackers might help patients monitor adherence to medications. There are a variety of alcohol and substance use trackers that might be useful as an adjunctive tool to help individuals discontinue substance misuse. Apps could also potentially be used as adjunctive tools to help psychiatric patients manage comorbid medical conditions, as it is not uncommon for individuals to experience physical complications due to their underlying psychiatric illness.

\section{Limitations}

Although the literature and our own informal investigations suggest that smartphone apps can be useful in mental healthcare, it is essential to recognise their limitations (Box 2). One of the major concerns about the use of smartphone apps in healthcare is data security and data protection,

B0X 2 Some limitations of mental health apps

- Apps are no match for a consultation with a mental health specialist

- Not all patients have access to smartphones and apps

- Current app developers are often not clinical specialists

- Patient data must be kept secure

- The technology can fail

- Many current apps lack rigorous evaluation of the evidence base, content, efficacy and safety

- Many current apps are not kept up to date after publication 
especially since there has been a recent push towards granting patients the right to access their electronic medical records. Another major concern is that smartphone apps may not be able to reach marginalised individuals in society. Given that many of these individuals do not have access to smartphones and apps, it is unlikely that these technologies could be applied universally as part of a treatment regimen or as an adjunctive tool. Thus, it is important to note that smartphone apps are likely to facilitate care, but it is unlikely that they would entirely replace the care provided. Also, it should be noted that an app in itself should never replace a professional consultation with a mental health specialist and this should be clearly stated in the disclaimer section of the app.

Despite the reported success of smartphone apps as an adjunct to standard psychotherapy for substance misuse (Rizvi 2011), there are two key limitations that should be noted. First, any psychological intervention delivered via a smartphone app is unlikely to replicate the clinical efficacy of face-to-face sessions with a therapist. Second, although people receiving community drug treatment (in the UK, at least) usually have mobile phones, many do not have access to smartphones and hence they might not benefit from the apps developed to help manage drug misuse (Milward 2015). In my opinion, addiction psychiatrists will probably find that smartphone apps are more likely to help in early detection rather than as an adjunct to conventional treatment.

Another drawback is the fact that the technology itself might not be reliable or it might fail. It is not uncommon for apps to become outdated, as

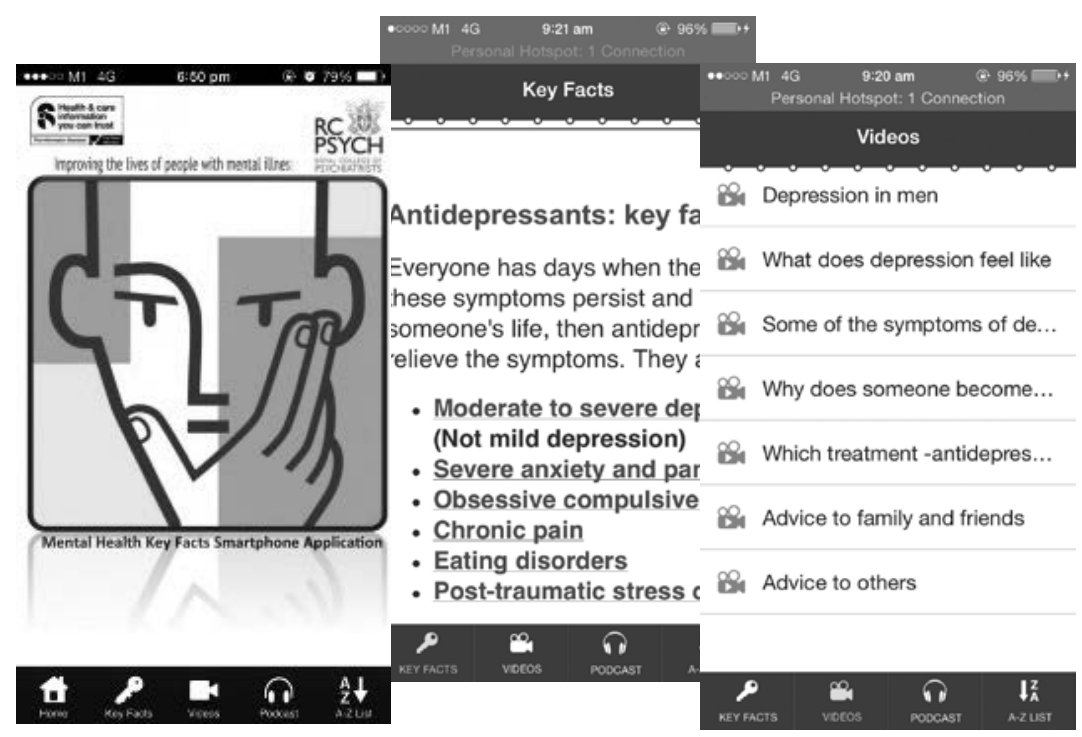

Screen shots from the Royal College of Psychiatrists' mental health app. The app is available for free from iTunes and Google Play Store. their developers do not have a sustainable business model to support continued development. A real concern pertains to the evidence base for particular apps and information on their standard and safety. Most of the commercially available apps come from developers with only limited clinical knowledge, and this compromises the information quality of these products as well as the evidence base on the smartphone app in healthcare.

\section{Future directions}

\section{Actions at an individual level}

\section{Direct involvement in app development}

Collaboration with technology experts in the creation and development of apps for patients is one way in which psychiatrists can take the lead in overcoming limitations. A more direct way is to equip psychiatrists with the basic technical skills to develop apps themselves - something we discussed in our previous article (Zhang 2015a). One such app is Mental Health Key Facts (Fig. 1), which provides an online handheld version of key facts information for patients produced by the Royal College of Psychiatrists (www.rcpsych.ac.uk/ healthadvice/moreinformation/newrcpsychapp. aspx). The app, designed and created by the first author (M.W.B.Z.), was launched during the College's International Congress in 2014 and has received a total of 346 unique downloads.

Most of the currently available mental health apps simply give information about psychiatric disorders. These apps have a limited lifespan and utility. If psychiatrists were more involved, especially by creating apps themselves, they could improve the quality and usefulness of apps for patients. For example, in considering the development of an app to help people with borderline personality disorder manage their condition, it is not adequate simply to provide information about their disorder. Psychological therapies could be added to the app, along with local helplines for individuals who are in crisis. Most alcohol trackers to date simply enable users to log their drinks and compute an average blood alcohol level. This is not useful for individuals who want to be empowered to take control of their drinking problem. Psychological tools such as functional behavioural analysis and setting behavioural goals could be integrated into tracker apps, to allow self-management of alcohol use. A recently published paper (Zhang 2015b) shows how this can be achieved if clinicians are involved. These are just a few examples of resources that could be added to mental health apps that would help patients manage their condition 
between consultations with their psychiatrist or psychologist.

Naturally, not all psychiatrists have the time and the willingness to embrace the technology for creating apps on their own. However, it is hoped that psychiatrists would at least be involved in the formulation and the scoping of apps in conjunction with the app developers.

\section{Actions at an organisational level}

Peer review of apps

With the advancements in smartphone technology and the limitations of the existing literature, we recommend that psychiatrists provide more frequent literature reviews of apps pertinent to their practice. As we explained in our article on educational apps for medical and psychiatry students (Zhang 2015a), such reviews would help to identify a collection of apps that have been useful in patient care. Psychiatrists should also conduct randomised controlled trials of patient apps (similar to the manner in which educational psychiatric apps have been reviewed) and publish the results. As randomised controlled trials usually take time to complete, a proof of concept or a feasibility study might be sufficient for an app that is based on conventional methodologies.

\section{Identification of a collection of safe apps}

Psychiatrists or healthcare organisations might also compile a list of peer-reviewed apps that are deemed appropriate for use by patients. Our previous article describes a self-certification model for app review that offers a systematic method for clinicians and healthcare professionals to determine whether an app is safe and applicable to their practice (Zhang 2015a). The model (Box 3) is based on the Health on the Net Foundation's code for assessing the reliability and credibility of the information on medical and health websites.

\section{Establishing and encouraging innovations}

E-volution is a unique initiative set up by the South West Division of the Royal College of Psychiatrists to share local innovations in psychiatry (RCPsych 2014). E-volution is an online forum open to all College members and affiliates that has been running as a 1-year pilot since 14 November 2014. The advantage of such a portal is that it inspires core and senior trainees to innovate, with the support of the local trust.

\section{Actions at a national level}

National organisations in the UK should spearhead the review of psychiatry apps. The NHS
B0X 3 Self-certification model for determining the information quality of apps

- The information presented in the app should be attributed to an author

- The purpose of the app and its intended audience should be defined

- Confidentiality requires that the app includes a privacy policy

- Information included should have a specific date of creation

- Claims regarding the benefits or performance of any treatments need to be supported by scientific evidence

- The developer's contact details must be included

- Disclosures (e.g. funding and conflict of interest) should be declared

- Any advertising in an app should have appropriate disclosure

(Based on Health on the Net Foundation 2008)

Choices Health Apps Library (http://apps.nhs. uk), which was initiated in December 2012, aims to accomplish this by identifying healthcare apps and having them reviewed by health professionals, app developers and the general public. Sustaining this rigour in app evaluation will result in a list of health-related apps deemed clinically safe and relevant to people living in England. To date, 17 mental health apps from the apps stores have been rated and deemed safe. A further 10 are listed but have not yet been rated. This collection may be useful for practising psychiatrists to recommend to their patients.

\section{Conclusions}

Over the past decade, there has been major advancement in smartphone technology, such that smartphones and apps are now playing an adjunctive role in patient care. It is hoped that psychiatrists, through our articles in Advances, will be more cognisant of the advantages and limitations of smartphones apps. Taking into consideration the limitations, psychiatrists might consider how best they could contribute to embracing and utilising this technology in the future at the individual, organisation and national levels.

\section{References}

BinDhim NF, Shaman AM, Trevena L, et al (2015) Depression screening via a smartphone application: cross-country user characteristics and feasibility. Journal of the American Medical Informatics Association, 22: $29-34$. 
Bsoul M, Minn H, Tamil L (2011) Apnea MedAssist: real-time sleep apnea monitor using single-lead EEG. IEEE Transactions on Information Technology in Biomedicine, 15: 416-27.

Edgar S, Swyka T, Fulk G, et al (2010) Wearable shoe-based device for rehabilitation of stroke patients. Conference Proceedings: Annual International Conference of the IEEE Engineering in Medicine and Biology Society: $3772-5$

Faurholt-Jepsen M, Frost M, Vinberg M, et al (2014) Smartphone data as objective measures of bipolar disorder symptoms. Psychiatry Research, 217: $124-7$

Grünerbl A, Muaremi A, Osmani V, et al (2015) Smart-phone based recognition of states and state changes in bipolar disorder patients. IEEE Journal of Biomedical and Health Informatics, 19: 140-8.

Health on the Net Foundation (2008) HONCode. HON (https://www. healthonnet.org/HONcode/Webmasters/intro.html).

Huang CC, Lee PY, Chen PY, et al (2012) Design and implementation of a smartphone-based portable ultrasound pulsed-wave Doppler device for blood flow measurement. IEEE Transactions on Ultrasonics, Ferroelectrics, and Frequency Control, 59: 182-9.

Marshall A Medvedev 0, Antonov A (2008) Use of smartphone for improved self-management of pulmonary rehabilitation. International Journal of Telemedicine and Applications, doi: 10.1155/2008/753064.

Milward J, Day E, Wadworth E, et al (2015) Mobile phone ownership usage and readiness to use by patients in drug treatment. Drug and Alcohol Dependence, 146: 111-5.

Mosa AS, Yoo I, Sheets L (2012) A systematic review of healthcare applications for smartphones. BMC Medical Informatics and Decision Making, 12: 67

Oresko JJ, Duschl H, Cheng AC (2010) A wearable smartphonebased platform for real-time cardiovascular disease detection via electrocardiogram processing. IEEE Transactions on Information Technology in Biomedicine, 14: 734-40.
Ozdalga E, Ozdalga A, Ahuja N (2012) The smartphone in medicine: a review of current and potential use among physicians and students. Journal of Medical Internet Research, 14: e128.

Palmier-Claus JE, Ainsworth J, Machin M, et al (2012) The feasibility and validity of ambulatory self-report of psychotic symptoms using a smartphone software application. BMC Psychiatry, 12: 172

Palmier-Claus JE, Rogers A, Ainsworth J, et al (2013) Integrating mobilephone based assessment for psychosis into people's everyday lives and clinical care: a qualitative study. BMC Psychiatry, 13: 34

Pramana G, Parmanto B, Kendall PC, et al (2014) The SmartCAT: an $\mathrm{m}$-health platform for ecological momentary intervention in child anxiety treatment Telemedicine Journal and e-Health, 20: 419-27.

Rizvi SL, Dimeff LA, Skutch J, et al (2011) A pilot study of the DBT coach: an interactive mobile phone application for individuals with borderline personality disorder and substance use disorder. Behaviour Therapy, 42 : 589-600.

Royal College of Psychiatrists (2014) Innovation in the South West Division: E-volution. RCPsych (http://www.rcpsych.ac.uk/ workinpsychiatry/divisions/southwest/innovationinthesouthwest.aspx). Accessed 12 June 2015

Sposaro F, Danielson J, Tyson G (2010) iWander: an android application for dementia patients. Conference Proceedings: Annual International Conference of the IEEE Engineering in Medicine and Biology Society: $3875-8$

Worringham C, Rojeck A, Stewart I (2011) Development and feasibility of a smartphone, ECG and GPS system for remotely monitoring exercise in cardiac rehabilitation. PLoS One, 6: e14669.

Zhang MWB, Ho CSH, Cheok CCS, et al (2015a) Bringing smartphone technology into undergraduate and postgraduate psychiatry. BJPsych Advances, 201: 222-8.

Zhang MW, Ho RC (2015b) The alcohol self-management smartphone application: an evidence-based approach. BMJ Innovations, 6 July, doi: 10.1136/bmjinnov-2015-000057 [Online First].

\section{MCOs}

Select the single best option for each question stem

\section{Smartphone apps are not currently used in} schizophrenia as:

a a tool for self-report of symptoms

b a tool to correlate symptoms with stressors

c a tool to detect core early relapse symptoms

d a diagnostic tool to help clinicians diagnose the illness

e a tool to enable psychiatrists understand patient's symptoms and stressors.

\section{Which of the following is not a limitation to} the use of smartphone apps for patients?

a data security and data protection

b reduced access to smartphone technology of marginalised members of the society

c potential failure of the technology

d applications becoming out of date

e the compromising of face-to-face psychotherapeutic interventions.
3 Which of the following is not one of the certification criteria for determining the information quality of apps?

a checking for the presence of a privacy policy or disclaimer

b checking for the presence of appropriate disclosures

c checking whether the purpose of the app has been defined

d checking whether claims are backed by scientific evidence

e checking the absolute number of downloads and peer reviewers' comments.

4 Which of the following has not been proposed as a future direction for psychiatric apps for patient care?

a actions at the individual level in developing evidence-based apps

b actions at the individual level in beta-testing other developers' apps c actions at the organisational level involving peer review of apps

$\mathrm{d}$ actions at the organisational level involving identification of a collection of safe healthcare apps

e actions at the national level in compiling a list of safe and trusted apps.

5 In medicine, smartphone technology has been used in patient care as:

a a tool for remote monitoring of patients

b a tool for self-management of chronic disease

c a medical device

$\mathrm{d}$ a diagnostic tool to help clinicians make medical diagnoses

e all of the above. 